Annuaire suisse de politique de développement

$17 \mid 1998$

Propriété intellectuelle : quels enjeux pour les pays en développement ?

\title{
8. Politique économique extérieure
}

\section{CpenEdition}

\section{Journals}

Édition électronique

URL : http://journals.openedition.org/aspd/764

DOI : 10.4000/aspd.764

ISSN : 1663-9669

Éditeur

Institut de hautes études internationales et du développement

Édition imprimée

Date de publication : 1 avril 1998

Pagination : 273-301

ISSN : 1660-5934

Référence électronique

«8. Politique économique extérieure », Annuaire suisse de politique de développement [En ligne], 17|

1998, mis en ligne le 31 juillet 2012, consulté le 08 septembre 2020. URL : http://

journals.openedition.org/aspd/764; DOI : https://doi.org/10.4000/aspd.764

(c) The Graduate Institute I Geneva 


\section{POLITIQUE ÉCONOMIQUE EXTÉRIEURE}

\subsection{GARANTIE CONTRE LES RISQUES À L'EXPORTATION (GRE)}

La GRE permet aux exportateurs suisses de se protéger contre le risque de ne pas être payé pour des livraisons. Les garanties couvrent essentiellement des livraisons dans les pays en développement. Fin 1996, le total des engagements de la GRE atteignait 5,9 milliards de francs (dont $63 \%$ pour des pays asiatiques). Les nouvelles garanties accordées en 1996 se sont élevées à 1,4 milliard de francs, soit un tiers de moins que l'année précédente. La Communauté de travail des æuvres d'entraide et la Déclaration de Berne ont mené depuis 1996 une campagne très active contre l'octroi de la GRE pour des livraisons de matériel destiné à la construction du barrage des Trois Gorges en Chine. Les auvres d'entraide ont aussi profité de cette campagne pour réitérer leurs demandes pour une réforme du fonctionnement de la GRE.

La Garantie suisse contre les risques à l'exportation (GRE) est un instrument que la Confédération met à la disposition de l'exportateur pour lui faciliter la conclusion de contrats à l'étranger. La GRE a été créée en 1934, pour favoriser l'emploi et promouvoir les exportations. Cet appui est très apprécié lorsque des difficultés de recouvrement sont à craindre en raison de la situation politiquement et économiquement instable dans le pays de l'acheteur. Une commission de huit membres nommés par le Conseil fédéral examine les demandes de garantie ${ }^{1}$. La commission de la GRE était présidée par l'ambassadeur Wilhelm B. Jaggi (OFAEE) jusqu'en 1997, lorsque l'ambassadeur Rolf Jeker lui succéda (il avait déjà assumé cette fonction de 1986 à 1991). En 1980, le Parlement a ajouté la disposition suivante à la loi sur la GRE (art. 1 ${ }^{\text {er }}$, al. 2): «S'agissant d'exportations à destination des pays en développement les plus défavorisés, la Suisse tiendra compte des principes fondamentaux de la politique suisse en matière d'aide au développement. " La plupart des garanties couvertes par la GRE concernent des livraisons dans les pays en développement.

Parmi les perspectives pour les années à venir, la Suisse va réviser l'ordonnance de 1989 fixant les primes. Les pays membres de l'arrangement de l'OCDE sur les crédits à l'exportation ont discuté des moyens de mieux harmoniser les systèmes nationaux de soutien public au financement des exportations. Les négociations de l'OCDE ont abouti le 20 juin 1997 à l'adoption d'un accord sur des lignes directrices concernant les primes minimales à payer pour des garanties. Les différences entre les soutiens publics de garantie sur des exportations peuvent représenter des distortions de la concurrence lors de l'attribution de marchés. L'accord prévoit des primes minimales tenant compte des risques qui existent dans le pays acheteur et qui doivent permettre l'autonomie financière des

1. En 1997, la commission comprenait quatre représentants de la Confédération (OFAEE, DDC, Administration des finances, OFIAMT), trois représentants de l'économie privée (Union suisse du commerce et de l'industrie - Vorort, Société suisse des constructeurs de machines, Société suisse des industries chimiques) et une représentante des milieux syndicaux (Syndicat chrétien de l'industrie, de l'artisanat et des services). 
institutions de GRE, pour obliger certains pays à augmenter leurs primes (et diminuer ainsi leur appui aux exportateurs). L'application de ces nouvelles règles entraînera pour la Suisse une adaptation des primes d'ici 1999. L'accord de l'OCDE de juin 1997 prévoit aussi une harmonisation de l'évaluation des risques que présentent les pays bénéficiaires de la GRE (classification commune des risques). L'accord entrera en vigueur le $1^{\mathrm{er}}$ avril 1999, au terme de la période transitoire d'adaptation des systèmes nationaux de GRE.

\section{RISQUES COUVERTS PAR LA GRE}

La GRE couvre l'exportateur contre certains risques encourus dans ses contrats avec l'étranger que ni luimême ni l'acheteur étranger ne sont en mesure d'influencer. II s'agit des risques suivants:

\begin{tabular}{ll}
\hline Risque politique & $\begin{array}{l}\text { Evénements politiques (guerre, révolution, émeutes...) qui empêchent } \\
\text { l'acheteur d'effectuer les paiements convenus. }\end{array}$ \\
\hline$\square$ Risque de transfert & $\begin{array}{l}\text { Les paiements de l'acheteur ne sont pas transférés en raison de mesures } \\
\text { gouvernementales de contrôle des changes ou de rééchelonnement de la } \\
\text { dette extérieure du pays. }\end{array}$ \\
\hline$\square$ Risque de fabrication & $\begin{array}{l}\text { Si une marchandise n'est pas livée suite à des événements politiques ou } \\
\text { à des mesures gouvernementales. }\end{array}$
\end{tabular}

La GRE ne couvre pas le risque d'insolvabilité d'un acheteur privé, ni les risques pouvant être couverts par des assurances privées (risques de transport par exemple), ni les risques monétaires ou les fluctuations de taux de change. La garantie contre les risques monétaires avait dû être supprimée en 1985, en raison des grandes pertes qu'elle avait provoquées.

Deux nouveautés depuis 1996:

\section{Risque de ducroire (risque commercial)}

Insolvabilité ou refus de payer de la part d'acheteurs de droit public ou de sociétés d'utilité publique. Sur demande de l'exportateur, ce risque peut maintenant aussi être couvert pour des acheteurs privés, lorsque ceux-ci bénéficient de la garantie de paiement d'une banque acceptée par la GRE ou de l'Etat (jusqu'en juillet 1996, seules les garanties de banques étatiques étaient acceptées).

- Risque secondaire des contrats libellés en monnaie étrangère

Nouveauté introduite dès juillet 1996, l'exportateur peut, contre paiement d'un supplément de prime, faire calculer l'indemnisation en francs suisses, selon le taux de change en vigueur au moment du sinistre. Ce n'est pas une garantie sur les taux de change. Cette couverture garantit uniquement que l'exportateur ne sera pas sous-assuré en raison de fluctuations de change. Lorsque le franc suisse baisse jusqu'au jour du sinistre, l'indemnité devient en effet insuffisante pour le refinancement en monnaie étrangère du crédit d'exportation.

Sources: Bureau pour la GRE, Zurich; L'Agefi, 12 septembre 1997. 
Le montant total des nouvelles garanties accordées s'est élevé à 1,4 milliard de francs, soit $34 \%$ de moins que l'année précédente (2,1 milliards). Le nombre des demandes déposées a pourtant augmenté de 186 en 1996, pour atteindre 708 demandes. Cette évolution à première vue surprenante s'explique toutefois par le fait que les demandes ont principalement augmenté pour des livraisons de moindre importance (moins d'un demi-million de francs), alors que le nombre des grands projets a baissé (plus de demandes de la part de petites et moyennes entreprises).

Les garanties accordées se concentrent essentiellement sur deux branches économiques. En 1996, l'industrie des machines a obtenu $63 \%$ des garanties, et l'industrie chimique $36 \%$. Les contrats assurés représentent une très petite part des exportations totales de la Suisse (1,4\% en 1996). La part des contrats couverts par la GRE peut par contre être prépondérante dans les exportations de la Suisse vers certains pays en développement (comme l'Algérie par exemple).

Tableau n ${ }^{0} 19$

Répartition géographique des nouvelles garanties

\begin{tabular}{|c|c|c|c|c|}
\hline \multirow[b]{2}{*}{ Groupes de pays } & \multicolumn{2}{|c|}{1995} & \multicolumn{2}{|c|}{1996} \\
\hline & $\begin{array}{r}\text { Millions } \\
\text { de fr. }\end{array}$ & $\begin{array}{l}\text { part } \\
\text { en } \%\end{array}$ & $\begin{array}{r}\text { Millions } \\
\text { de fr. }\end{array}$ & $\begin{array}{l}\text { part } \\
\text { en \% }\end{array}$ \\
\hline Europe (sans Europe cent. et orient.) & 195.1 & 9.1 & 201.2 & 14.1 \\
\hline Pays d'Europe centrale et orientale & 61.8 & 2.9 & 43.5 & 3.1 \\
\hline Amérique centrale et du Sud & 160.5 & 7.5 & 155.4 & 10.9 \\
\hline Afrique & 121.3 & 5.6 & 170.0 & 11.9 \\
\hline Asie & 1515.6 & 70.5 & 854.2 & 60.0 \\
\hline Australie & 95.0 & 4.4 & 0.0 & 0.0 \\
\hline Total & 2'149.3 & 100.0 & 1 '424.3 & 100.0 \\
\hline dont: 72 pays les plus défavorisés ${ }^{1}$ & 868.1 & 40.4 & 166.4 & 11.7 \\
\hline dont: Amérique & 0.0 & 0.0 & 1.5 & 0.1 \\
\hline Afrique & 85.5 & 4.0 & 10.9 & 0.8 \\
\hline Asie & 782.6 & 36.4 & 154.0 & 10.8 \\
\hline
\end{tabular}

1. Les «pays les plus défavorisés» définis par la GRE comprennent les PMA, ainsi que les autres pays en développement dont le PNB par tête était inférieur à 765 dollars par habitant en 1995 (voir liste en note 4). Le nombre de pays compris dans la liste des pays défavorisés a passé de 64 en 1995 à 72 en 1996. L'Indonésie et l'Egypte ont été retirées de cette liste en 1996. Les chiffres de 1996 ne peuvent donc pas être comparés avec ceux des années précédentes.

Source: Rapport annuel 1996 de la GRE - Juin 1997, tableaux des pages 11 et 13.

Les données sur la répartition géographique des nouvelles garanties accordées (tableau n ${ }^{\circ} 19$ ci-dessus) montrent qu'en 1996, comme les années précédentes, la majorité des nouveaux contrats ont concerné l'Asie $(60 \%$ des nouvelles garanties); $14 \%$ des nouvelles garanties ont ensuite concerné l'Europe (principalement la Turquie). La part de l'Afrique est de 12\% (majorité des nouvelles garan- 
ties pour l'Egypte, l'Algérie et, dans une moindre mesure, l'Afrique du Sud). Les exportations à destination de l'Amérique latine ont bénéficié de $11 \%$ des garanties. Les $70 \%$ de ces nouvelles garanties concernent le Mexique, le Brésil et le Venezuela. La part des garanties octroyées pour des livraisons dans les pays de l'Europe centrale et orientale est faible, mais certains de ces pays bénéficient du système de garantie de crédits à l'exportation prévu dans le crédit de programme pour la coopération avec les pays de l'Europe centrale et orientale et les pays de la Communauté des Etats indépendants (voir chapitre 10 de la partie «Revue» de cet Annuaire). La baisse très forte des nouvelles garanties pour des livraisons à destination des pays les plus défavorisés s'explique par un changement de classification des pays (voir plus bas le sous-chapitre sur ces pays les plus défavorisés).

\section{$\square$ Total des engagements de la GRE}

La baisse des engagements totaux observée depuis une quinzaine d'années s'est poursuivie en 1996 (-6,7\% de 1995 à 1996). Le total des engagements s'élevait à 5,9 milliards de francs fin 1996 . Le tableau n ${ }^{\circ} 20$ plus loin montre la répartition géographique des engagements de la GRE et le graphique suivant concerne les 15 principaux pays bénéficiaires. Ces 15 pays représentent les $79 \%$ du total des engagements de la GRE. Notons que c'est la première fois que le Bureau pour la GRE publie, dans le Rapport annuel 1996, les données statistiques détaillées des engagements de la GRE par pays.

\section{Graphique $\mathbf{n}^{\circ} 3$ \\ Principaux pays couverts par la GRE \\ Montant total des engagements au 31 décembre 1996, en millions de francs}

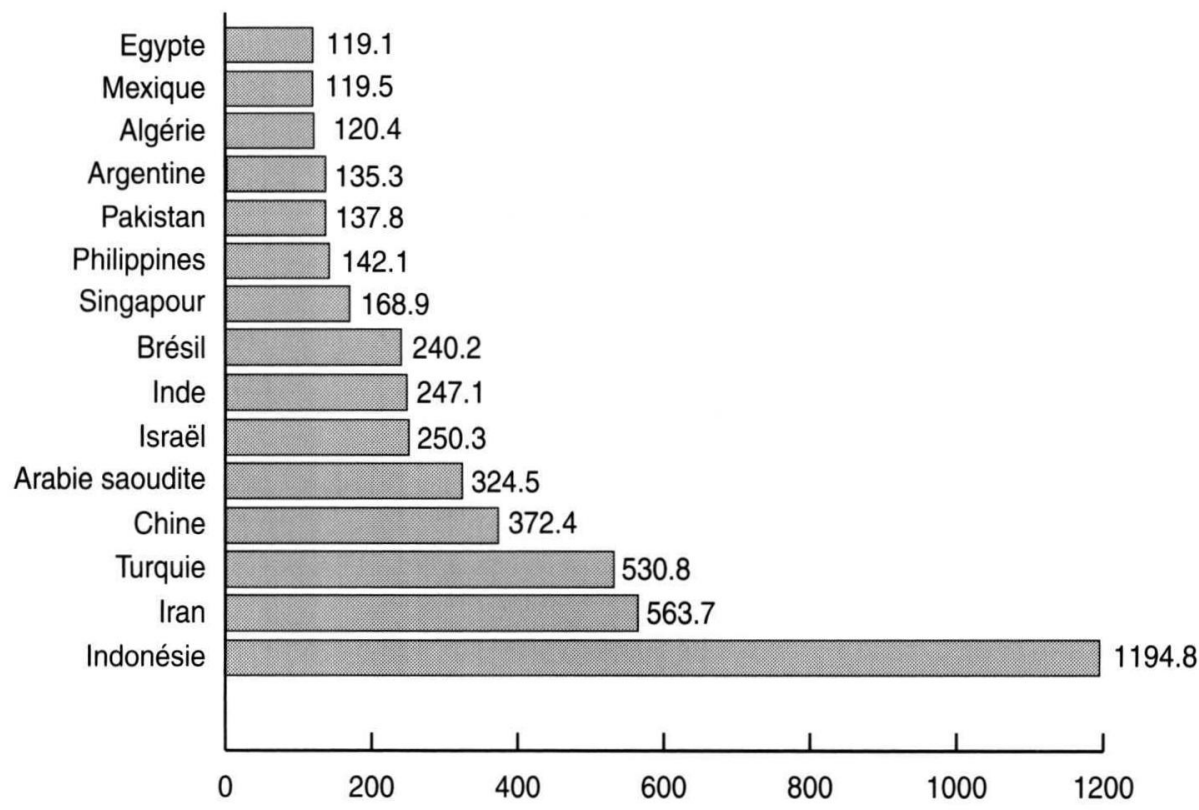

Source: Rapport annuel 1996 de la GRE - Juin 1997, à partir des tableaux de l'annexe G. 
Tableau n $\mathbf{n}^{\circ} \mathbf{2 0}$

Répartition géographique du total des engagements

\begin{tabular}{|c|c|c|c|c|c|c|}
\hline \multirow[b]{2}{*}{ Groupes de pays } & \multicolumn{3}{|c|}{1995} & \multicolumn{3}{|c|}{1996} \\
\hline & $\begin{array}{l}\text { millions } \\
\text { de fr. }\end{array}$ & $\begin{array}{l}\text { part } \\
\text { en \% }\end{array}$ & $\begin{array}{l}\text { différence } \\
\text { avec } 1994 \\
\text { en } \%\end{array}$ & $\begin{array}{l}\text { millions } \\
\text { de fr. }\end{array}$ & $\begin{array}{l}\text { part } \\
\text { en \% }\end{array}$ & $\begin{array}{c}\text { différence } \\
\text { avec } 1995 \\
\text { en \% }\end{array}$ \\
\hline $\begin{array}{l}\text { Europe (sans Europe } \\
\text { centrale et orientale) }\end{array}$ & 825.7 & 13.0 & -12.4 & 693.1 & 11.7 & -16.1 \\
\hline $\begin{array}{l}\text { Pays d'Europe centrale } \\
\text { et orientale }\end{array}$ & 222.5 & 3.5 & -11.4 & 158.3 & 2.7 & -28.9 \\
\hline $\begin{array}{l}\text { Amérique centrale et } \\
\text { du Sud }\end{array}$ & 950.4 & 14.9 & -14.9 & 729.6 & 12.3 & -23.2 \\
\hline Afrique & 599.7 & 9.4 & -32.8 & 528.9 & 8.9 & -1.8 \\
\hline Asie & 3673.3 & 57.7 & 9.2 & 3746.1 & 63.1 & 2.0 \\
\hline Australie & 95.0 & 1.5 & & 84.5 & 1.4 & -11.1 \\
\hline Total & 6366.6 & 100.0 & -3.6 & 5940.5 & 100.0 & -6.7 \\
\hline $\begin{array}{l}\text { dont: } 72 \text { pays } \\
\text { les plus défavorisés }\end{array}$ & 2330.4 & 36.6 & 0.8 & 941.8 & 15.9 & -59.6 \\
\hline dont: Europe centr. $e$ & & 0.0 & & 0.4 & 0.0 & \\
\hline Amérique & 2.3 & 0.0 & -20.7 & 10.7 & 0.2 & 365.2 \\
\hline Afrique & 280.7 & 4.4 & -24.8 & 146.2 & 2.5 & -47.9 \\
\hline Asie & 2047.4 & 32.2 & 5.7 & 784.5 & 13.2 & -61.7 \\
\hline
\end{tabular}

Source: Rapport annuel 1996 de la GRE - Juin 1997, tableaux des pages 11 et 13.

Les caractéristiques principales de la répartition géographique des engagements de la GRE sont les suivantes (situation fin 1996):

- Asie

La part relative de l'Asie a continué de croître, pour atteindre plus de $63 \%$ de toutes les garanties. Les parts de toutes les autres régions ont baissé. Neuf pays sur les 15 principaux pays couverts par la GRE sont des pays asiatiques (10 pays avec la Turquie, classée dans le continent européen). L'Indonésie est de loin le principal pays couvert par la GRE (près d'un tiers de toutes les garanties en Asie). La plus grande partie des garanties concerne le groupe $\mathrm{ABB}$, impliqué dans trois grands projets énergétiques ${ }^{2}$.

Malgré l'engagement très important de la GRE dans certains pays asiatiques et la crise économique sévère dans les pays d'Asie du Sud-Est, la commission de la GRE se veut rassurante. Selon les déclarations faites en janvier 1998, la GRE est à même de faire face à une éventuelle augmentation de l'incapacité de payer de certains Etats. Un défaut de paiement d'un pays tel que l'Indonésie coûterait annuellement environ 100 millions de francs à la GRE³ .

2. Journal de Genève, 13 janvier 1998 «La GRE porte un regard anxieux sur l'Asie».

3. Voir note précédente. 


\section{- Amérique centrale et du Sud}

Représente les $12 \%$ de tous les engagements, surtout pour le Brésil, l'Argentine, le Mexique et la Colombie (l'ensemble de ces pays représentant les $78 \%$ de toutes les garanties pour ce continent).

\section{口 Europe}

Représente aussi les $12 \%$ de tous les engagements, principalement pour la Turquie (531 millions de francs), la Grèce (100 millions de francs) et, dans une moindre mesure, le Portugal (28 millions) et la Slovénie (20 millions).

\section{- Afrique}

Seuls $9 \%$ des garanties couvertes pour les exportations suisses l'étaient pour le continent africain (pour plus de la moitié en faveur des pays de l'Afrique du Nord). Les principaux pays couverts par la GRE étaient l'Algérie (120 millions de francs), l'Egypte, l'Afrique du Sud, le Zimbabwe, le Cameroun et la Côte d'Ivoire (l'ensemble de ces pays représentant les $82 \%$ de toutes les garanties pour ce continent).

\section{- Europe centrale et orientale}

Ne représente que $3 \%$ des engagements de la GRE. Principalement pour couvrir des exportations vers la Roumanie, la Fédération de Russie, la République tchèque et la Slovaquie.

\section{$\square$ Pays en développement les plus défavorisés}

Pour la définition de ce groupe de pays, la Confédération se fonde sur une liste établie par le Comité d'aide au développement de l'OCDE. Cette liste est plus large que le groupe des pays les moins avancés (PMA) et inclut les pays à bas revenus dont le PNB par habitant était inférieur à 765 dollars par habitant en 19954. La liste des pays les plus défavorisés a été modifiée en 1996 (pour comprendre notamment des pays de l'Asie centrale de la Communauté des Etats indépendants). La liste comprend actuellement 72 pays, contre 64 en 1995. Il faut tenir compte de ce changement lorsque l'on compare les données de 1996 avec celles des années précédentes, et l'évolution de 1995 à 1996 dans les tableaux $n^{\text {os }} 19$ et 20 est ainsi difficile à interpréter. L'Egypte et l'Indonésie ont en effet été retirées de la liste (alors que les montants de la GRE apparaissent encore dans ce groupe de pays en 1995). Les nouvelles garanties pour l'Indonésie s'élevaient à 506 millions de francs en 1995 et le montant total des engagements à 1,065 milliard de francs (respectivement 56 et 125 millions de francs pour l'Egypte).

L'évolution des montants de la GRE entre 1996 et les années précédentes ne peut pas se faire en raison du changement du nombre de pays compris dans la liste des pays les plus défavorisés. En 1996, $93 \%$ des nouvelles garanties pour les pays les plus défavorisés concernaient le continent asiatique. Le total des engagements de la GRE pour ces pays atteignait 942 millions de francs en 1996, soit 16\% du total des engagements pour tous les pays; $83 \%$ du total des engagements pour ce groupe de pays concernait l'Asie (15,5\% l'Afrique, $1 \%$ l'Amérique latine).

4. La liste des 72 pays les plus défavorisés comprend les 48 pays les moins avancés (PMA) ainsi que les pays suivants: Albanie, Arménie, Azerbaidjan, Bosnie, Cameroun, Chine, Congo, Côte d'Ivoire, Géorgie, Ghana, Guyane, Honduras, Inde, Kenya, Kirghizistan, Mongolie, Nicaragua, Nigéria, Pakistan, Sénégal, Sri Lanka, Tadjikistan, Vietnam, Zimbabwe 


\section{- Versements d'indemnités en 1996}

Le montant total des indemnisations s'est élevé à 108,5 millions de francs en 1996 (pour 13 pays), soit 55 millions de moins qu'en 1995. Les principaux versements concernaient l'Algérie (36,9 millions de francs), la Fédération de Russie (36,6 millions), l'Egypte (9,7 millions), le Cameroun (5,8 millions), la Jordanie (5,6 millions) et l'Angola (3,8 millions). La majeure partie des versements d'indemnités (96 millions de francs) s'est effectuée en prévision d'accords de consolidation de dettes conclus entre la Confédération et les pays suivants: Algérie, Bulgarie, Cameroun, Egypte, Fédération de Russie, Honduras, Jordanie et Macédoine 5 .

\section{$\square$ Comptes de la GRE}

La politique et les conditions de garantie de la GRE sont soumises à l'obligation légale d'aboutir à terme à l'autonomie financière. La GRE a bouclé son exercice 1996 avec un résultat positif (22,9 millions de francs), cela pour la première fois depuis vingt ans. Après avoir procédé à un premier remboursement de 50 millions de francs à la Confédération en 1995, la GRE a réussi à rembourser une nouvelle tranche de 138 millions de francs sur les avances de la Confédération (avance cumulée de 1,59 milliard de francs fin 1996).

\section{$\square$ Consolidations et remises de dettes}

Fin 1996, une centaine d'accords bilatéraux de rééchelonnement de dettes commerciales étaient en cours dans une trentaine de pays. Les avoirs de la GRE issus de ces accords ont atteint 3,1 milliards de francs (intérêts capitalisés et intérêts échus, mais non payés, compris) ${ }^{6}$.

La Confédération a conclu en 1996 des accords bilatéraux de réduction ou de rééchelonnement de la dette avec huit pays: Algérie*, Cameroun, CongoBrazzaville, Croatie*, Gabon*, Honduras, Macédoine*, Sierra Leone (*rééchelonnement de la dette à un taux d'intérêt commercial). Le montant total rééchelonné s'élève à 158,8 millions de francs. Le rapport annuel de la GRE relève que la volonté et la capacité de paiement des pays débiteurs s'améliorent. En 1996, $82 \%$ des remboursements en capital dus ont été ainsi effectivement payés (61\% en 1995), ainsi que $84 \%$ des intérêts demandés (67\% en 1995).

\section{$\square$ Les $œ u v r e s$ d'entraide réclament une réforme de la GRE}

La Communauté de travail des œuvres d'entraide, la Déclaration de Berne et des organisations écologistes dénoncent régulièrement l'octroi de la GRE à certains projets qui ne respectent pas les objectifs de la coopération au développement ou du développement durable. La GRE a assuré des exportations pour de nombreuses centrales hydrauliques, dont certains projets étaient controversés (dont Atatürk et Karakaya en Turquie, Chixoy au Guatemala, El Cajon au Honduras, El Guario en Colombie, Itaipú au Brésil, Tarbela au Pakistan). La GRE n'a par contre pas participé au projet controversé du barrage de Bakun en Malaisie.

5. La liste complète des versements d'indemnités figure à l'annexe C du Rapport annuel 1996 de la GRE.

6. Rapport annuel 1996 de la GRE. L'annexe D contient la liste de tous les accords de consolidation en cours conclus depuis 1974. Les paiements effectués par la GRE (y compris les intérêts capitalisés) se sont élevés à 3,593 milliards de francs, les remboursements des pays débiteurs à 580 millions de francs (part de la GRE) et les intérêts échus, mais non payés au 31 décembre 1996, à 117 millions. 
Le barrage de Manantali au Mali avait été construit de 1982 à 1988. Les entreprises Losinger et les Ateliers de Vevey avaient participé aux travaux et le Conseil fédéral avait accordé une garantie de 155 millions de francs. Le bilan après la mise en service du barrage est selon la Déclaration de Berne très mitigé: moins de surfaces irriguées que prévu, explosion de la malaria et de la bilharziose, navigabilité pas améliorée. Aucune électricité n'a été produite, puisque la centrale électrique n'a pas encore vu le jour faute de financement (projet actuellement remis à l'ordre du jour par la Banque mondiale). La Déclaration de Berne et Greenpeace demandent à la Suisse de s'opposer à ce projet de la banque s'il se fait au détriment de l'agriculture traditionnelle sur les berges du lac.

Les campagnes de la Déclaration de Berne en 1996 et 1997 et celle menée par la Communauté de travail des œuvres d'entraide se sont focalisées d'une part sur l'opposition à l'octroi de la GRE aux livraisons suisses pour la construction du barrage des Trois Gorges (voir ci-dessous); d'autre part, elles réclament aussi une réforme du fonctionnement de la GRE (prise en compte des objectifs de développement durable et des droits de l'Homme dans l'octroi de la GRE, élargissement de la commission de la GRE à un représentant des œuvres d'entraide et meilleure transparence sur les garanties accordées).

Comme indiqué au début de ce texte, la Suisse doit tenir compte des principes fondamentaux de la politique suisse en matière d'aide au développement lorsqu'elle accorde la garantie à un projet. Un seul projet (au Lesotho) aurait été refusé sur la base de cet article? ${ }^{7}$ Dans le texte des Lignes directrices Nord-Sud, le Conseil fédéral reconnaissait que des conflits d'intérêts pouvaient exister entre la politique de développement et la politique de promotion des exportations: «Dans l'appréciation des risques en vue de l'octroi de la garantie aux risques à l'exportation pour des livraisons dans les pays en développement les plus pauvres, il est nécessaire de mettre plus d'accent sur les aspects d'ordre politique et sur le respect des droits de l'Homme dans les pays récipiendaires.» ${ }^{8}$

Pour mieux tenir compte des objectifs de développement, la Communauté de travail a fait de nouveau pression sur le Conseil fédéral pour que - comme dit plus haut - la commission de la GRE soit élargie pour comprendre un représentant des œuvres d'entraide (revendication d'une campagne de lettres adressées au conseiller fédéral Jean-Pascal Delamuraz au printemps 1997). Cette demande réitérée depuis de nombreuses années n'a pas encore été acceptée par la Confédération. Le Conseiller fédéral a cependant proposé d'examiner la création d'une sous-commission consultative pour les aspects de politique de développement (composée par des représentants de l'économie privée, de l'administration fédérale et d'organisations non gouvernementales).

La Communauté de travail et la Déclaration de Berne relèvent aussi depuis de nombreuses années le manque de transparence sur les demandes d'octroi de la GRE pour des projets particuliers, alors qu'une garantie publique devrait donner droit à un débat public. La commission de la GRE et les milieux économiques évoquent souvent le secret commercial pour ne pas dévoiler la liste des projets d'entreprises demandant la couverture par la GRE. L'examen des projets et l'évaluation des risques doivent, selon l'avis de la Société des constructeurs de

7. Déclaration de Berne, Vers un développement solidaire, «Le barrage de la démesure - Campagne contre la participation suisse au barrage chinois des Trois Gorges», Déclaration de Berne, numéro spécial 140, août 1997.

8. Voir texte des Lignes directrices Nord-Sud, Annuaire Suisse Tiers Monde 1995, pp. 218-231. 
machines, se faire à l'abri des pressions intérieures et de l'étranger'. Les entreprises ont droit au secret commercial et ne fourniraient pas certaines informations confidentielles si le débat était public. La commission tient compte des objectifs de politique de développement, notamment avec la présence d'un représentant de la DDC, et n'a pas besoin d'un contrôle par des organisations extérieures.

\section{$\square$ Barrage des Trois Gorges sur le fleuve Yangtsé en Chine}

La Chine, très dépendante de l'énergie tirée du charbon (centrales thermiques au charbon très polluantes), cherche à augmenter sa production d'électricité. Le barrage des Trois-Gorges doit permettre de fournir de l'électricité, mais aussi de prévenir les inondations et d'améliorer les conditions de navigation sur le fleuve Yangtsé. Les travaux sont en cours et, début novembre 1997, le cours du fleuve était détourné par un canal pour permettre la construction du barrage principal qui devrait être achevé en 2009.

Production d'électricité: c'est la construction du barrage le plus puissant au monde, avec 26 générateurs produisant 18'200 mégawatts (MW) au total. Pour comparaison, le plus puissant barrage du monde en service, Itaipú au Brésil, a un potentiel de 12'600 MW.

Dimension du barrage: une longueur de 2335 mètres et une hauteur de 185 mètres. Ce n'est pas le plus haut barrage du monde, puisqu'une dizaine d'autres barrages présentent une hauteur supérieure, dont la Grande-Dixence (285 mètres) et Mauvoisin en Suisse. Le lac de retenue aura une longueur de 600 kilomètres et une superficie de 1084 kilomètres carrés (soit deux fois le lac de Constance).

Coût du projet: officiellement évalué à 26,6 milliards de dollars en 1993, ce coût pourrait selon certaines sources tripler pour atteindre 75 milliards de dollars.

La Communauté de travail des œuvres d'entraide et la Déclaration de Berne (en collaboration avec le WWF et Greenpeace) ont mené depuis 1995 une campagne très active contre ce projet de barrage. Elles se sont opposées à l'octroi de la GRE à ce projet. Des critiques à l'encontre du projet de barrage se sont élevées en Chine (scientifiques et experts, membres du Congrès et autorités régionales) et sur le plan international (Etats-Unis et Japon par exemple, ainsi que des organisations de défense de l'environnement). Les critiques adressées par la Déclaration de Berne et la Communauté de travail des œuvres d'entraide à ce projet comprennent les points suivants:

- Le barrage monumental des Trois Gorges est une mauvaise réponse aux besoins énergétiques de la Chine. La Chine devrait investir plus dans la promotion d'énergies alternatives et encourager les économies d'énergie dans les installations industrielles désuètes qui gaspillent beaucoup d'énergie. La construction décentralisée de plusieurs barrages plus modestes pourrait être une solution de rechange qui réduirait l'impact sur l'environnement.

- Le barrage est une réponse très partielle au problème des inondations, car il ne permettra pas d'éviter les crues d'importants affluents du fleuve Yangtsé en aval du barrage. Une rupture du barrage menacerait 200 millions de personnes (risque sismique).

9. Martin Erb, directeur de la Société suisse des constructeurs de machines, in Neue Zürcher Zeitung, 6 décembre 1996. 
- Alors que la coopération suisse accorde une grande importance à la participation des populations concernées par un projet de développement, la Suisse ne doit pas cautionner un projet qui est imposé à la population locale (manque de débat public) et qui entraînera le déplacement forcé de 1,8 million de personnes. Une telle évacuation ne pourra pas se faire sans résistances, perspective inquiétante pour un pays réputé pour ses violations des droits de l'Homme et la répression des mouvements d'opposition. Un projet d'une telle envergure pourrait d'ailleurs difficilement se faire dans un pays démocratique, en raison des oppositions. Le Conseil fédéral ne devrait donc pas soutenir un projet qui serait «impensable» en Suisse. Le lac de retenue engloutira une douzaine de villes, des milliers de villages, des centaines d'industries et des centaines de sites culturels, historiques et archéologiques.

- Les conséquences écologiques de la construction du barrage des Trois Gorges sont incalculables: des dizaines de milliers d'hectares de terres fertiles engloutis sous les eaux; faune, forêts et flore détruites par le lac de retenue en amont du barrage; vie de la faune sous-marine perturbée et certaines espèces animales menacées; augmentation du taux de salinité des rivières suite à l'augmentation de l'évaporation. Le volume du lac de retenue risque en outre de diminuer rapidement par l'accumulation de dépôts de sédiments.

Deux entreprises (ABB Asea Brown Boveri et Sulzer) avaient répondu à un appel d'offres international lancé par les autorités chinoises. L'offre d'ABB portait sur une commande de 14 générateurs pour un montant de 340 millions de francs. Sulzer espérait pouvoir exporter des éléments de turbines pour 25 millions de francs. La commission de la GRE, puis le Conseil fédéral (en décembre 1996) avaient donné un préavis favorable aux demandes de principe déposées par les deux entreprises pour la couverture des exportations par la GRE (les représentants de la DDC et des syndicats s'étaient opposés à l'octroi de la garantie). L'accord définitif devait être confirmé par la commission de la GRE et le Département fédéral de l'économie publique si la Chine commandait effectivement le matériel proposé. En août 1997, les autorités chinoises ont décidé d'attribuer une partie des contrats pour la centrale électrique du barrage à l'entreprise ABB pour le montant de 340 millions de francs (l'offre de Sulzer n'a pas été retenue). Le soutien de la GRE a effectivement été confirmé par le Conseil fédéral à la fin de l'année 1997. La direction d'ABB estime que l'octroi de la GRE était une condition indispensable pour pouvoir répondre à l'appel d'offres international concernant ce barrage des Trois Gorges, puisque les Chinois ont privilégié les fournisseurs bénéficiant d'une garantie publique. ABB invoque aussi comme argument la sauvegarde d'emplois en Suisse.

La Déclaration de Berne avait lancé fin 1996 un appel au Conseil fédéral pour lui demander de ne pas accorder de garantie à ce projet (avec l'appui de 330 personnalités suisses). Elle a ensuite lancé le 4 août 1997 une campagne contre le barrage des Trois Gorges et pour des réformes de la GRE. La campagne s'est notamment appuyée sur l'opinion publique, puisque des cartes postales ont été envoyées à $\mathrm{ABB}$ et au Conseil fédéral. Les cartes postales demandaient à $\mathrm{ABB}$ de renoncer à participer à la réalisation du barrage et celles adressées au conseiller fédéral Jean-Pascal Delamuraz lui demandaient de renoncer à confirmer l'octroi de la GRE si ABB décrochait le contrat. 


\section{ABB ASEA BROWN BOVERI}

E GROUPE helvético-suédois ABB Asea Brown Boveri est l'une des grandes entreprises à demander régu-lièrement la GRE. Le chiffre d'affaires d'ABB s'élevait à 34,6 milliards de francs en 1996 (dont 872 millions seulement en Suisse). ABB réalise les $46 \%$ de son chiffre d'affaires dans le domaine de la production et la distribution d'énergie; $40 \%$ du chiffre d'affaires concernent les équipements industriels (secteurs pétrolier et pétrochimique, gaz, robots industriels, appareils électriques, etc.).

L'entreprise ABB est en pleine phase de restructuration, avec une forte diminution du nombre des places de travail en Europe et aux Etats-Unis, ainsi qu'une délocalisation dans les marchés émergents, essentiellement en Asie. En octobre 1997, ABB réaffirmait qu'elle entendait accélérer son implantation sur les marchés émergents (avec parallèlement une diminution de 10'000 emplois en Europe et aux Etats-Unis). L'entreprise employait 215'000 personnes dans le monde en 1996, dont 11'350 en Suisse et 26'200 en Suède. Parmi les pays en développement, ABB possède des filiales importantes dans les pays suivants: Afrique du Sud, Arabie Saoudite, Argentine, Brésil, Chine, Colombie, Corée du Sud, Egypte, Hongkong, Inde, Indonésie, Malaisie, Mexique, Philippines, Singapour, Taiwan, Thaïlande, Turquie, Venezuela et Vietnam. Parmi ces pays, ABB emploie du personnel surtout en Thaïlande (près de 6000 employés), au Brésil (plus de 4800), en Chine (3560) et en Afrique du Sud (2885). ABB a aussi augmenté sa présence dans des pays de l'Europe centrale et orientale (Pologne, République tchèque et Russie). En 1996, les principaux nouveaux investissements de l'entreprise ont concerné l'Afrique du Sud, la Bulgarie et la Chine. D'autres investissements ont aussi été entrepris au Mozambique, en Namibie et au Sénégal.

Source: Rapport de gestion 1996 - Groupe ABB et sociétés mères, 1997.

\section{SOURCES}

Sur le fonctionnement général de la GRE

Bureau pour la GRE, OFAEE, ERG, Rapport annuel 1996 de la GRE - Juin 1997, 1997.

OFAEE, Communiqué de presse, 26 juin 1997, «Les pays de l'OCDE conviennent de lignes directrices relatives aux primes de la GRE».

Schweizerischer Handels- und Industrie-Verein (Vorort), Wirtschaftspolitisches Jahrbuch 1996, 1997, S. 37-40.

Richard Gerster, in Swiss Coalition News, «Official Export Credits and Development Policy: The Case of Switzerland», Nr 13, September 1997.

L'Agefi, 26 août 1997 «GRE - Les primes vont baisser», 12 septembre 1997 «La GRE devra évoluer pour répondre aux défis qui se présenteront demain».

La Vie économique - Revue de politique économique, novembre 1997.

Neue Zürcher Zeitung, 29. November 1996 «Reformbedürftige Exportrisikogarantie» (Richard Gerster), 6. Dezember 1996 «Reformdürftige Exportrisikogarantie?» (Martin Erb), 2. Juli 1997 «OECD-Richtlinien über ERG-Prämien».

Bureau pour la garantie contre les risques à l'exportation, Kirchenweg 8, 8032 Zurich.

Sur le projet du barrage des Trois Gorges

Dossier de presse de la Déclaration de Berne, conférence de presse du 4 août 1997, Berne.

Communauté de travail des œuvres d'entraide, Communiqués de presse, Berne, 18 septembre 1997.

Déclaration de Berne, Vers un développement solidaire, «Le barrage de la démesure - Campagne contre la participation suisse au barrage chinois des Trois Gorges», Déclaration de Berne, numéro spécial 140, août 1997.

EvB Dokumentation, «Vom Verstummen der Flüsse», Erklärung von Bern, Dokumentation 2/1997.

EvB-Magazin 3a/1997, «Die Schweiz unter Wasser?».

L'Agefi, 25 août 1997 «ABB parmi les fournisseurs du barrage des Trois Gorges».

Le Courrier, 8 août 1997 «La campagne contre la participation suisse au projet des Trois Gorges est lancée».

Journal de Genève, 1“-2 février 1997 «Les barrages en eaux troubles», 27 juin 1997 «Les écologistes suisses ne veulent toujours pas d'une centrale électrique au Mali», 5 août 1997 «Le barrage chinois des Trois Gorges suscite une controverse planétaire », 8-9 novembre 1997 «Le projet des Trois Gorges franchit un cap décisif».

Le Monde, 2 avril 1997, 10 novembre 1997 «La dérivation du Yangtzé, étape décisive vers le plus grand barrage du globe".

Neue Zürcher Zeitung, 19.-20. April 1997 «Hydropolitik gegen Öko-Fundamentalismus - Grosse Staudämme als Zankapfel der Entwicklungs- und Umweltpolitik», 10. November 1997 «Umleitung des Jangtse in den Drei Schluchten».

La Liberté, 5 août 1997 «Les opposants au barrage des Trois Gorges battent le rappel».

\section{SITE INTERNET}

Déclaration de Berne: http://www. access. ch/evb/db 


\title{
8.2. PRÉFÉRENCES DOUANIÈRES
}

La Suisse accorde depuis 1972 des droits de douane préférentiels sur l'importation de certains produits en provenance de pays en développement. En septembre 1996, le Parlement a accepté la révision du schéma de préférences. Sur la base de cette révision, l'ordonnance fixant les droits de douane préférentiels est entrée en vigueur le $1^{\text {er }}$ mars 1997. La nouvelle ordonnance prévoit une série d'améliorations de l'accès au marché suisse. Les préférences tarifaires sont cependant supprimées dès 1998 pour une douzaine de pays en développement économiquement avancés.

Le schéma de préférences douanières appliqué par la Suisse est un instrument de la politique économique extérieure qui a pour but de promouvoir l'intégration des pays en développement dans le commerce international. Le système généralisé des préférences (SGP) établi par la CNUCED permet aux pays industrialisés d'adopter des schémas nationaux donnant aux pays en développement un accès préférentiel aux marchés du Nord, en baissant les droits de douane sur certaines importations en provenance de ces pays (voir encadré sur le SGP).

\section{SYSTÈME GÉNÉRALISÉ DES PRÉEÉRENCES (SGP)}

\begin{abstract}
E CONCEPT des préférences douanières a été discuté à la CNUCED lors de sa première conférence en
1964. L'objectif principal était l'accélération de la croissance économique des pays en développement en augmentant leurs exportations et en favorisant leur industrialisation. Un modèle uniforme avec des concessions tarifaires valables pour tous les pays étant difficile à adopter en raison des différences dans la protection douanière entre les pays, la CNUCED II en 1968 a établi le système généralisé des préférences, en fixant des objectifs et des principes communs. Ce système est un ensemble d'une quinzaine de schémas nationaux (dont un schéma unique appliqué par tous les pays de I'Union européenne). Le SGP constitue une dérogation au principe de I'OMC de la nation la plus favorisée. Les schémas de préférences sont des mesures unilatérales (les pays industrialisés ne demandent pas la réciprocité pour leurs exportations vers les pays en développement) et autonomes (préférences octroyées par les pays industrialisés sans négociations avec les pays bénéficiaires). Des exonérations supplémentaires sont accordées aux PMA.
\end{abstract}

\section{$\square$ Politique suisse de préférences tarifaires}

La Suisse a appliqué des préférences douanières au profit des pays en développement depuis 1972. Le schéma a été révisé en 1981 et prolongé en 1991. L'efficacité des préférences douanières pour promouvoir les exportations des pays en développement s'est avérée très limitée, ceci pour plusieurs raisons ${ }^{10}$.

Premièrement, suite aux négociations du Tokyo Round et de l'Uruguay Round (GATT/OMC), les tarifs douaniers ont sensiblement baissé, ce qui provoque une érosion de la marge préférentielle qui peut être accordée par les schémas de préférences. Les droits de douane moyens appliqués par les pays industrialisés aux produits finis s'élèvent à $4 \%$ après l'Uruguay Round.

10. Pour les études sur l'efficacité du système de préférences douanières, voir le «Message sur la prorogation de l'arrêté fédéral sur les préférences tarifaires", ou aussi l'étude de Marc Bacchetta in Effets de l'Uruguay Round sur les pays en développement, Genève, IUED/LEA/Ecodiagnostic, 1995, vol. V. 
Deuxièmement, la gestion administrative peut être complexe pour les exportateurs des pays du Sud qui désirent profiter du schéma de préférences. La multiplicité des schémas de préférences d'un pays à l'autre ne facilite pas la tâche de l'exportateur (coût administratif). Il doit en outre prouver l'origine du produit (règles d'origine), ce qui est plus difficile à faire pour un produit industriel (utilisant beaucoup de produits semi-finis) que pour un produit agricole.

Troisièmement, les droits de douane ne sont qu'un obstacle au commerce parmi d'autres (restrictions quantitatives, barrières techniques, etc.).

Le tableau $n^{\circ} 21$ (page suivante) présente les montants des importations de la Suisse ayant bénéficié des préférences ces dernières années. Trois quarts environ des importations en provenance des pays en développement pourraient bénéficier des préférences (soit $6 \%$ environ du volume total des importations de la Suisse). Le recours effectif à ces préférences est relativement faible, puisqu'en moyenne seule la moitié des avantages tarifaires qui pourraient être accordés est effectivement utilisée. Ce degré d'utilisation des préférences a régulièrement augmenté de 1988 à 1994, en passant de 36\% à 52\%, pour ensuite rester aux alentours de ce niveau. Le degré d'utilisation des avantages accordés est en fait très élevé pour les produits agricoles, alors qu'il est plus bas que la moyenne pour les produits industriels (la marge de préférence étant faible pour certains produits industriels, les exportateurs hésitent à faire les démarches nécessaires qui leur permettraient de bénéficier de ces avantages).

La meilleure utilisation du schéma s'est traduite par une diminution croissante du produit des droits de douane, le calcul étant effectué sur la base du tarif normal. En 1992, la diminution des droits de douane accordée grâce aux préférences ainsi calculée se montait à près de 70 millions de francs suisses, pour s'élever à 83 millions de francs en 1994 et atteindre 100,5 millions en 1997. Les concessions tarifaires bénéficient surtout à un petit nombre de pays. La Chine et l'Inde ont bénéficié de $45 \%$ de ces concessions. Les autres pays principaux bénéficiaires du schéma de préférences étaient ces dernières années la Thaillande, la Corée du Sud, le Brésil, l'Indonésie, la Malaisie et le Pakistan. La part des importations suisses sous le schéma de préférences en provenance des PMA est minime (diminution des droits de douane pour les PMA de 4,3 millions de francs seulement sur le total de 100,5 millions en 1997 pour l'ensemble des pays bénéficiaires du schéma suisse).

\section{$\square$ Prolongation de l'application du schéma suisse de préférences tarifaires}

Le Conseil fédéral a présenté en mai 1996 son message sur la prorogation de dix ans de l'arrêté fédéral sur les préférences tarifaires. Malgré l'efficacité limitée des préférences douanières, le Parlement a décidé de reconduire son schéma, en le ciblant mieux sur les intérêts des pays les moins avancés (PMA). Des mesures complémentaires de promotion du commerce sont aussi prises dans le crédit de programme sur la continuation du financement des mesures de politique économique et commerciale (crédit adopté en 1996). Si la part des pays en développement dans le commerce mondial est en augmentation ces dernières années, la réalité est très différenciée selon les régions, avec une forte expansion du commerce extérieur de certains pays asiatiques, alors que la part des PMA dans le commerce mondial a baissé de 0,6 à $0,3 \%$ en dix ans. 
Tableau $\mathbf{n}^{\circ} 21$

Importations suisses et préférences tarifaires 1994-1996

\begin{tabular}{lccr}
\hline Importations et préférences & $\mathbf{1 9 9 4}$ & $\mathbf{1 9 9 5}$ & $\mathbf{1 9 9 6}$ \\
\hline $\begin{array}{l}\text { 1. } \\
\text { Volume total des importations } \\
\text { de la Suisse, en millions de francs }\end{array}$ & 92608 & 94483 & 96664 \\
\hline $\begin{array}{l}\text { Importations en provenance } \\
\text { des pays en voie de développement } \\
\text { (PVD), en millions de francs }\end{array}$ & 6426 & 6175 & 5828 \\
\hline $\begin{array}{l}\text { Part des importations en } \\
\text { provenance des PVD en pour-cent } \\
\text { (point 2 en pour-cent du point 1) }\end{array}$ & $6.9 \%$ & $6.5 \%$ & $6.0 \%$ \\
\hline $\begin{array}{l}\text { Importations pouvant bénéficier } \\
\text { du traitement préférentiel, } \\
\text { en millions de francs }\end{array}$ & 4471 & 4223 & 4326 \\
\hline $\begin{array}{l}\text { Importations ayant effectivement } \\
\text { bénéficié des préférences, } \\
\text { en millions de francs }\end{array}$ & 2319 & 2188 & 2204 \\
\hline $\begin{array}{l}\text { Degré d'utilisation des avantages } \\
\text { accordés en pour-cent } \\
\text { (point } 5 \text { en pour-cent du point 4) }\end{array}$ & $52 \%$ & $52 \%$ & $51 \%$ \\
\hline
\end{tabular}

Source: OFAEE.

\section{- Produits concernés par le schéma de préférences}

D'importantes concessions ayant déjà été faites dans le secteur industriel, la révision se concentre surtout sur des améliorations spécifiques dans le domaine agricole. Le Conseil fédéral traite les pays en développement selon les mêmes conditions que celles offertes aux Etats de la zone de libre-échange européenne. Même s'il reconnaît que les pays les moins avancés ne concurrencent que marginalement la production agricole suisse, les importations en provenance de ces pays restent soumises au système de contingentement pour les produits qui sont aussi récoltés en Suisse (tarifs douaniers très élevés selon la saison).

\section{- Règles d'origine}

«Les règles d'origine doivent garantir que seuls les produits entièrement obtenus ou ayant subi une transformation ou un traitement suffisants dans un pays en développement bénéficiaire puissent obtenir un traitement préférentiel à l'importation en Suisse. ${ }^{11}$ Une nouvelle ordonnance relative aux règles d'origine est entrée en vigueur le $1^{\text {er }}$ juillet 1996. Une nouvelle règle permet d'utiliser des matériaux ou produits semi-finis d'origine suisse et de les considérer comme originaires du pays en développement concerné dans le cadre de la détermination de l'origine. Cette mesure devrait favoriser la coopération industrielle entre entreprises suisses et celles des pays bénéficiaires du schéma de préférences. Cette mesure sera dans le futur étendue aux matériaux et produits semi-finis en provenance de l'Union européenne.

ASTM 97, «Préférences douanières», pp. 127-128. 


\section{- Principe de la graduation}

Nouveauté du nouveau schéma de préférences tarifaires, la Suisse va appliquer le principe de «graduation intégrale» selon le stade de développement atteint par le pays partenaire. C'est-à-dire qu'elle va supprimer toutes les préférences accordées aux pays en développement les plus avancés. Le choix des pays qui seront gradués se fera notamment sur la base de la liste des pays en développement avancés fixée par l'OCDE (pays qui ne sont plus dans la liste des pays bénéficiaires de l'aide publique au développement, mais dont l'aide est comptabilisée dans un agrégat séparé d'aide publique, comme les pays en transition les plus avancés). La Suisse retire dès le $1^{\text {er }}$ mars 1998 les préférences tarifaires à certains pays en développement: le Mexique, maintenant membre de l'OCDE, et tous les pays en développement avec lesquels la Suisse a conclu des accords de libreéchange.

Le principe de «graduation partielle» permet en outre à la Suisse de supprimer les préférences douanières pour quelques produits exportés par certains pays. La Suisse a déjà fait usage de cette possibilité dans les schémas précédents, en n'octroyant pas de préférences tarifaires pour les textiles en provenance de la Chine, de la Corée, de Hongkong et de Macao. Certains produits ne bénéficient que de préférences réduites (avantages de 30\% seulement pour les montres en provenance de Hongkong par exemple). Le Conseil fédéral considère que les préférences douanières sont une aide au décollage et qu'il est possible de retirer les préférences sur des produits particulièrement compétitifs pour lesquels un pays est capable d'affronter le marché international.

\section{- Autres mesures}

Une clause de sauvegarde permet de supprimer des préférences tarifaires si les intérêts de la Suisse sont menacés pour un produit ou un secteur donné. Cette clause n'a encore jamais été utilisée par la Suisse.

Les importateurs de certains produits doivent cotiser à des fonds de garantie pour financer des stocks obligatoires (de café par exemple). Afin d'inciter les importateurs à s'approvisionner dans les PMA, le Conseil fédéral a la compétence d'autoriser le remboursement aux importateurs des contributions versées au titre des stocks obligatoires. Les dépenses de la Confédération pour cette mesure ne devraient cependant pas dépasser les recettes supplémentaires engendrées par le retrait des préférences aux pays les plus avancés.

\section{$\square$ Procédure de consultation informelle et adoption par le Parlement}

Une douzaine d'organisations se sont prononcées dans le cadre de la consultation informelle menée par l'OFAEE. Plusieurs organisations économiques se sont exprimées contre des concessions supplémentaires dans les domaines des textiles, de l'habillement et du tabac.

La Communauté de travail des œuvres d'entraide a pris position sur le projet de révision du schéma de préférences tarifaires, en estimant que le projet n'allait pas assez loin. La révision devrait permettre d'offrir bien davantage aux PMA plutôt que de retirer les préférences tarifaires aux pays les plus avancés. Elle critique notamment le retrait des préférences à un pays simplement sur la base de sa qualité de membre de l'OCDE (Mexique). Le système de graduation ne se justifierait que dans la mesure où les concessions retirées sont compensées par de 
substantielles améliorations pour les PMA et autres pays à faible revenu. La Communauté de travail regrette que la Suisse pénalise, par la graduation partielle, des secteurs concurrentiels dans certains pays en développement. Elle estime qu'une telle mesure peut être arbitraire et protectionniste, alors qu'une clause générale de sauvegarde existe déjà lorsqu'une importation menace vraiment un secteur économique en Suisse. La croissance économique de certains pays est quelquefois très dépendante d'un secteur particulièrement performant (effet d'entraînement pour d'autres secteurs). La Communauté de travail souhaite aussi que la Suisse renonce à la progressivité des droits de douane en fonction du degré de transformation des produits, même dans le domaine des textiles (secteur important pour certains pays en développement). Elle regrette aussi que certaines préférences tarifaires ne soient accordées que dans la limite de contingents agricoles. Le Conseil fédéral devrait en outre examiner les moyens d'accorder des préférences dans d'autres secteurs (services, investissements, propriété intellectuelle).

\section{CLAUSES SOCIALES ET ENVIRONNEMENTALES}

D ANS SON "Message sur la prorogation de l'arrêté fédéral urgent sur les préférences tarifaires", le Conseil fédéral estime qu'il est inadapté de prévoir, par sanction, le retrait des préférences tarifaires en cas de violation de normes sociales (en raison, entre autres, du problème de contrôle, difficile à effectuer). Il pourrait par contre examiner plus tard les moyens d'appliquer un système de bonus (clauses d'incitation) dans le cas où l'Union européenne instaure un tel système. La Communauté de travail des œuvres d'entraide souhaitait que la Suisse prenne l'initiative et introduise des préférences supplémentaires pour les pays respectant des normes sociales et environnementales reconnues au niveau international (normes de l'OIT sur le plan social à appliquer dans un premier temps). Plusieurs organisations économiques ont exprimé leur doute quant à l'opportunité d'introduire de telles clauses. Lors du débat au Conseil national, une minorité de la commission de politique extérieure proposait l'introduction d'un article stipulant que le Conseil fédéral peut octroyer des préférences supplémentaires pour les importations de produits qui sont fabriqués en tenant compte des aspects sociaux ou en respectant l'environnement (proposition rejetée par 84 voix contre 35).

Le Conseil des Etats et le Conseil national ont adopté sans opposition la prorogation de dix ans de l'arrêté fédéral sur les préférences tarifaires en faveur des pays en développement en septembre 1996.

\section{$\square$ Ordonnance fixant les droits de douane préférentiels}

La révision de l'ordonnance, entrée en vigueur le $1^{\text {er }}$ mars 1997, constitue la première étape dans la mise en œuvre de l'arrêté fédéral adopté par les Chambres fédérales. L'ordonnance privilégie les pays les moins avancés, en leur accordant une exemption totale des droits de douane pour leurs produits industriels et la franchise de douane pour la plupart de leurs produits agricoles, y compris les produits agricoles transformés. Conformément au principe de la graduation, les pays qui ont atteint un degré élevé de développement sont exclus du schéma dès le $1^{\text {er }}$ mars 1998, dont le Mexique, membre de $1^{\prime} O C D E^{12}$. Les autres pays en

12. La liste des pays qui sont exclus du schéma de préférences à partir du $1^{\text {er }}$ mars 1998 comprend les pays suivants. Europe: Chypre. Asie: Brunei, Corée du Sud, Emirats arabes unis, Hongkong, Koweït, Qatar, Singapour. Amérique: Bahamas, Bermudes, îles Caïmans et Falkland, Mexique. 
développement seront mis presque sur pied d'égalité avec les partenaires de libre-échange de la Suisse. Le schéma de préférences tarifaires s'applique en outre à certains pays de l'Europe centrale et orientale, de même qu'à certains Etats de la $\mathrm{CEI}^{13}$.

Avec le principe de la graduation partielle, quelques pays asiatiques n'ont pas de préférences tarifaires pour certains produits (textiles, chaussures, jouets, meubles) ou bénéficient de préférences réduites (montres, parapluies). Pour d'autres produits, l'importation n'est exempte de droits de douane que dans la limite d'un contingent déterminé (fèves de soja, arachides, coprah, huiles de soja, d'arachide, de tournesol et de coco).

\section{SOURCES}

«Message sur la prorogation de l'arrêté fédéral sur les préférences tarifaires» du 29 mai 1996, Berne, in Feuille fédérale, $\mathrm{n}^{\circ} 28$, vol. III, 16 juillet 1996, pp. 153-178 (message 96.045).

Ordonnance fixant les droits de douane préférentiels en faveur des pays en développement, Berne, 29 janvier 1997.

Bulletin de l'Assemblée fédérale, session d'automne 1996, Berne (Conseil des Etats, 18 septembre 1996; Conseil national, 25 septembre 1996).

Rules of Origin of the Swiss GSP Scheme, Berne, OFAEE, 1997.

OFAEE, Communiqué de presse, 30 janvier 1997, «Produits en provenance des pays en développement: amélioration des conditions d'importation".

OFAEE, Berne (données relatives aux préférences douanières de 1994 à 1996).

Communauté de travail des œuvres d'entraide, dossier de presse, Berne.

\subsection{DIPLOMATIE COMMERCIALE ET ACCORDS ÉCONOMIQUES BILATÉRAUX (PROTECTION DES INVESTISSEMENTS, DOUBLE IMPOSITION)}

L'économie de la Suisse dépend fortement des relations commerciales et financières avec l'extérieur. Les conditions-cadres favorisant les investissements et la libre circulation des capitaux sont d'une grande importance. A côté de sa participation dans les organisations multilatérales, la Confédération accorde une grande importance à la conclusion d'accords bilatéraux visant à intensifier les relations commerciales, à ouvrir de nouveaux marchés et à accroître les investissements à l'étranger. La diplomatie commerciale comprend les visites de délégations de représentants de l'administration fédérale et de l'économie privée. Les négociations avec de nombreux pays partenaires ont abouti à la création d'un réseau dense d'accords bilatéraux pour promouvoir et protéger les investissements et pour éviter de pénaliser les entreprises et investisseurs par la double imposition dans le pays d'origine et le pays d'accueil des capitaux. La participation d'entreprises suisses à des foires économiques permet aussi, surtout aux PME, de faire connaître les produits (technologies, machines, etc.) que la Suisse peut exporter. Les accords de promotion et de protection des investissements prévoient la non-discrimination des entreprises de l'autre partie à l'accord et le libre transfert des revenus et autres montants afférents aux investissements. Les mesures éventuelles d'expropriation ou de nationalisation doivent donner lieu à des indemnisations et les différends peuvent être soumis à un tribunal arbitral.

13. Les pays suivants sont inclus dans la liste des bénéficiaires des préférences douanières: Etats issus de l'exYougoslavie, Albanie, Etats de la CEI en Asie centrale, Arménie, Azerbaïdjan, Géorgie, Moldavie. 


\section{$\square$ Accords de protection des investissements}

A défaut d'un cadre juridique multilatéral suffisamment développé, les pays industrialisés ont conclu depuis une trentaine d'années des accords bilatéraux avec les partenaires économiques. La Suisse fait partie des pays qui ont tissé un réseau très dense d'accords bilatéraux concernant la protection et l'encouragement des investissements. La Confédération souhaite ainsi assurer à ses entreprises une sécurité juridique accrue quand elles investissent à l'étranger. Fin novembre 1997, la Suisse avait signé 86 accords ( 73 sont en vigueur). Le tableau $\mathrm{n}^{\circ} 22$ page suivante donne la liste des accords conclus avec des pays en développement et des pays d'Europe centrale et orientale.

La protection des investissements englobe toutes les catégories d'avoirs dans l'autre pays: propriété de biens et d'immeubles, actions, parts sociales et autres formes de participation dans des sociétés, les concessions, droits d'auteur, droits de propriété industrielle (brevets, dessins ou modèles industriels, marques, noms commerciaux). Chaque partie contractante s'engage à encourager, dans la mesure du possible, les investissements de l'autre partie contractante. Les principes de la nation la plus favorisée et de non-discrimination doivent être respectés. Aucun pays ne peut donc soumettre sur son territoire les investissements de l'autre pays à un traitement moins favorable que celui qu'il accorde aux investisseurs de tout Etat tiers (sauf à de rares exceptions, notamment lorsque le pays fait partie d'une zone de libre-échange). Le libre transfert des revenus des investissements doit être garanti (intérêts, dividendes, apports supplémentaires de capitaux pour le développement des investissements, produits de la liquidation partielle ou totale d'un investissement). Des mesures éventuelles d'expropriation ou de nationalisation doivent donner lieu au paiement d'indemnités adéquates. Les différends entre les parties contractantes ou entre une partie et un investisseur de l'autre partie donnent lieu à des consultations et peuvent être soumis à un tribunal arbitral.

Un arrêté fédéral délègue au Conseil fédéral la compétence de signer des accords de protection des investissements, sans qu'il soit donc nécessaire au Parlement d'approuver chaque accord signé entre la Suisse et un pays tiers ${ }^{14}$.

\section{$\square$ Accords de double imposition}

Les pays signataires d'une convention de double imposition abandonnent une partie de leur souveraineté en matière fiscale afin d'éviter que des revenus soient imposés doublement, dans les deux pays concernés. Ces conventions bilatérales règlent l'imposition des personnes physiques et des entreprises: revenus, revenus immobiliers, dividendes, intérêts de créances, redevances, gains en capital, revenus d'artistes et sportifs, pensions. Dans le cas où l'autre pays contractant ne perçoit pas d'impôts sur la fortune, la convention ne porte que sur les impôts sur les revenus. L'élimination de la double imposition est dans l'intérêt des contribuables, mais assure aussi une protection fiscale aux entreprises suisses actives à l'étranger. Ces conventions garantissent en effet à ces entreprises de ne pas subir des conditions fiscales plus désavantageuses que celles dont bénéficient les entreprises concurrentes provenant d'autres Etats.

14. Le contenu général des accords de protection et de promotion des investissements est détaillé dans le document suivant: «Message sur la prorogation de l'arrêté fédéral concernant la conclusion de traités relatifs à la protection et à l'encouragement des investissements de capitaux» du 20 octobre 1993 (message 93.086) 
Tableau n 22

Liste des accords concernant la protection et la promotion des investissements (état au 30 novembre 1997)

Accords avec les pays en développement, les pays d'Europe centrale et orientale, et les pays de la CEI

\begin{tabular}{|c|c|c|c|c|c|}
\hline & $\begin{array}{c}\text { Année de la } \\
\text { signature }\end{array}$ & $\begin{array}{l}\text { Année de } \\
\text { l'entrée } \\
\text { en vigueur }\end{array}$ & & $\begin{array}{l}\text { Année de la } \\
\text { signature }\end{array}$ & $\begin{array}{l}\text { Année de } \\
\text { l'entrée } \\
\text { en vigueur }\end{array}$ \\
\hline \multicolumn{6}{|l|}{ Afrique } \\
\hline Tunisie & 1961 & 1964 & Gabon (dénoncé) ${ }^{1}$ & 1972 & 1972 \\
\hline Niger $^{1}$ & 1962 & 1962 & Zaïre & 1972 & 1973 \\
\hline Guinée $^{1}$ & 1962 & 1963 & Rep. centrafricaine ${ }^{1}$ & 1973 & 1973 \\
\hline Côte d'Ivoire' & 1962 & 1962 & Egypte & 1973 & 1974 \\
\hline Sénégal ${ }^{1}$ & 1962 & 1964 & Soudan & 1974 & 1974 \\
\hline Congo $^{1}$ & 1962 & 1964 & Mauritanie $^{1}$ & 1976 & 1978 \\
\hline Cameroun $^{1}$ & 1963 & 1964 & Mali & 1978 & 1978 \\
\hline Liberia $^{1}$ & 1963 & 1964 & Maroc & 1985 & 1991 \\
\hline Rwanda $^{1}$ & 1963 & 1963 & Ghana & 1991 & 1993 \\
\hline Togo ${ }^{1}$ & 1964 & 1966 & Cap-Vert & 1991 & 1992 \\
\hline Madagascar $^{1}$ & 1964 & 1966 & Gambie & 1993 & 1994 \\
\hline Tanzanie & 1965 & 1965 & Namibie & 1994 & - \\
\hline Bénin $^{1}$ & 1966 & 1973 & Zambie & 1994 & 1995 \\
\hline Tchad $^{1}$ & 1967 & 1967 & Afrique du Sud & 1995 & - \\
\hline Burkina-Faso' $^{1}$ & 1969 & 1969 & Zimbabwe & 1996 & - \\
\hline Ouganda & 1971 & 1972 & & & \\
\hline
\end{tabular}

\begin{tabular}{lcclcc}
\hline Amérique latine & & & & & \\
\hline Costa Rica & 1965 & 1966 & Paraguay & 1992 & 1992 \\
\hline Equateur & 1968 & 1969 & Honduras & 1993 & - \\
\hline Panama & 1983 & 1985 & Venezuela & 1993 & 1994 \\
\hline Bolivie & 1987 & 1991 & Brésil & 1994 & - \\
\hline Uruguay & 1988 & 1991 & El Salvador & 1994 & 1996 \\
\hline Jamaïque & 1990 & 1991 & Barbade & 1995 & 1995 \\
\hline Argentine & 1991 & 1992 & Mexique & 1995 & 1996 \\
\hline Chili & 1991 & - & Cuba & 1996 & 1997 \\
\hline Pérou & 1991 & 1993 & & &
\end{tabular}

1. Accords contenant également des dispositions relatives au commerce et à la coopération. 


\begin{tabular}{lcclccc}
\hline & Signature & Entrée en vigueur & & \multicolumn{2}{c}{ Signature } & Entrée en vigueur \\
\hline Asie & & & & & \\
\hline Corée du Sud & 1971 & 1971 & Vietnam & 1992 & 1992 \\
\hline Indonésie & 1974 & 1976 & Hongkong & 1993 & 1994 \\
\hline Jordanie & 1976 & 1977 & Pakistan & 1995 & 1996 \\
\hline Syrie & 1977 & 1978 & Cambodge & 1996 & - \\
\hline Malaisie & 1978 & 1978 & Laos & 1996 & 1996 \\
\hline Singapour & 1978 & 1978 & Mongolie & 1997 & - \\
\hline Sri Lanka & 1981 & 1982 & Philippines & 1997 & - \\
\hline Chine & 1986 & 1987 & Inde & 1997 & - \\
\hline Turquie & 1988 & 1990 & Thaïlande & 1997 & - \\
\hline
\end{tabular}

\section{Europe}

Malte 1965 1965

\section{Europe de l'Est et ex-URSS}

\begin{tabular}{lllllc}
\hline Hongrie & 1988 & 1989 & Ousbékistan & 1993 & 1993 \\
\hline Pologne & 1989 & 1990 & Biélorussie & 1993 & 1994 \\
\hline Tchéquie & 1990 & 1991 & Roumanie & 1993 & 1994 \\
\hline Slovaquie & 1990 & 1991 & Kazakhstan & 1994 & - \\
\hline Russie & 1990 & 1991 & Ukraine & 1995 & 1997 \\
\hline Bulgarie & 1991 & 1993 & Slovénie & 1995 & 1997 \\
\hline Albanie & 1992 & 1993 & Moldova & 1995 & 1996 \\
\hline Estonie & 1992 & 1993 & Macédoine & 1996 & 1997 \\
\hline Lettonie & 1992 & 1993 & Croatie & 1996 & - \\
\hline Lituanie & 1992 & 1993 & & & \\
\hline
\end{tabular}

Source: OFAEE.

Les négociations pour préparer une nouvelle convention peuvent être longues. Une fois paraphée, puis signée par les deux Etats (souvent lors d'une visite d'un conseiller fédéral dans ce pays), la convention doit être approuvée par le Parlement (sur la base d'un message du Conseil fédéral présenté aux Chambres fédérales $\left.{ }^{15}\right)$. La décision n'est pas soumise au référendum facultatif. La convention entre généralement en vigueur au début de l'année fiscale qui suit l'échange des instruments de ratification entre les deux Etats.

15. Les messages du Conseil fédéral demandant aux Chambres fédérales d'approuver la ratification de conventions de double imposition sont publiés dans la Feuille fédérale (voir liste des messages récents dans la liste des sources). Documents à commander à l'Office central des imprimés et du matériel, EDMZ, 3000 Berne. 


\section{LISTE DES PAYS AVEC LESQUELS LA SUISSE A SIGNÉ UNE CONVENTION DE DOUBLE IMPOSITION}

En italique, les conventions les plus récentes, signées en 1996 ou 1997

Pays en développement

Argentine, Chine, Corée du Sud, Côte d'Ivoire, Egypte, Equateur, Indonésie, Jamaïque, Malaisie, Maroc, Mexique, Pakistan, Singapour, Sri Lanka, Thaïlande, Trinité-et-Tobago, Tunisie, Venezuela, Vietnam.

Pays d'Europe centrale et orientale, pays de la CEI

Bulgarie, Fédération de Russie, Hongrie, Pologne, République slovaque, République tchèque, Roumanie, Slovénie.

Relativement peu de conventions ont été signées avec des pays de l'Amérique latine jusqu'en 1995. Seules les conventions avec le Mexique et l'Equateur (en 1994) avaient abouti, alors que les négociations avec d'autres pays furent longues. Jusqu'à la fin des années 80 , le Venezuela par exemple restait lié par les recommandations du Pacte andin, dont le modèle de convention reposait sur le principe de l'imposition dans l'Etat de la source du revenu. Cela allait à l'encontre de la politique conventionnelle suisse qui repose sur le modèle proposé par l'OCDE (plus favorable aux pays d'origine des investissements). La politique économique et financière plus libérale adoptée par le Venezuela ces dernières années sous la pression du FMI a rendu possible une négociation alignée sur la politique suivie par les pays industrialisés. C'est ainsi que la convention entre la Suisse et le Venezuela a pu être signée fin 1996, conformément aux vœux de la Suisse. Cette ouverture économique plus grande dans ce continent, la reprise de la croissance et la meilleure stabilité politique sont aussi des facteurs qui expliquent la signature en avril 1997 de la convention entre la Suisse et l'Argentine (dont les premiers pourparlers remontent à 1979).

\section{$\square$ Accords de libre-échange}

L'Association européenne de libre-échange (AELE) ne comprend depuis 1995 plus que les pays suivants: Islande, Liechtenstein, Norvège et Suisse. L'AELE a conclu une douzaine d'accords de libre-échange avec des pays tiers, avec pour objectif d'offrir aux entreprises exportatrices vers ces pays les mêmes conditions que celles dont bénéficient les entreprises de l'Union européenne. Les accords prévoient le libre-échange des produits industriels et des produits agricoles transformés. Ils comprennent aussi des règles de protection de la propriété intellectuelle et concernent également les marchés publics.

Des accords de libre-échange ont été conclus entre l'AELE et les pays suivants (état en décembre 1997): Bulgarie, Estonie, Hongrie, Israël, Lettonie, Lituanie, Pologne, Roumanie, Slovaquie, Slovénie, Tchéquie et Turquie. Des accords sont en préparation avec le Maroc et la Tunisie. Les accords avec les pays Baltes (Estonie, Lettonie et Lituanie) ont été acceptés par les Chambres fédérales en 1997. Ces accords ont permis de rendre multilatéraux des accords autrefois conclus bilatéralement entre la Suisse et ces pays. 
$\square$ Diplomatie commerciale, accords bilatéraux récents

(Asie, Amérique latine, Afrique, Europe centrale et orientale)

\section{Asie}

Dans l'ensemble des pays en développement, l'Asie est le continent avec lequel la Suisse a le plus de relations économiques. Plus des trois quarts des exportations de la Suisse vers le Tiers Monde se dirigent vers le continent asiatique et $68 \%$ des importations proviennent de ce continent ${ }^{16}$. Certains pays asiatiques figurent parmi les plus importants clients d'exportations suisses dans les pays en développement. En 1996, les principaux marchés d'exportation de la Suisse en Asie étaient le Japon, Hongkong (volume d'exportation de 2,7 milliards de francs), Singapour (1,4 milliard), Israël (1,2 milliard), la Corée du Sud (1,2 milliard), la Turquie, Taiwan, la Thaïlande et la Chine. L'Inde est le dixième pays partenaire de la Suisse.

Les visites de conseillers fédéraux ont été fréquentes pendant l'année passée sous revue. Le conseiller fédéral Jean-Pascal Delamuraz a entrepris à la tête de délégations économiques un voyage du 5 au 14 octobre 1996 au Japon, en Chine, au Cambodge et en Thaillande. Il s'est rendu du 30 mars au 5 avril 1997 aux Philippines et en Inde, puis en Turquie au mois de mai. Kaspar Villiger s'est rendu du 5 au 13 mai 1997 dans les pays d'Asie centrale (Azerbaïdjan, Kirghizistan, Turkménistan et Ouzbékistan. Flavio Cotti est allé avec des représentants de l'économie suisse au Vietnam, au Japon, en Indonésie et à Singapour du $1^{\text {er }}$ au 10 novembre. Le président de la Confédération, Arnold Koller, a fait une tournée au Népal, au Vietnam (Sommet de la francophonie) et en Thaïlande du 8 au 17 novembre. Treize pays asiatiques se trouvent parmi les 15 principaux marchés d'exportation de la Suisse (dans le groupe des pays en développement).

\section{口 Inde}

La visite en Inde de Jean-Pascal Delamuraz au début d'avril 1997 a débouché sur la signature d'une convention pour éviter la double imposition des sociétés et sur celle d'un accord pour la protection des investissements (pas encore en vigueur fin novembre 1997). Le Conseiller fédéral était accompagné d'une importante délégation des milieux économiques. Les discussions n'ont par contre pas permis de trouver un terrain d'entente concernant les barrières à l'importation de produits textiles ou horlogers en Inde, que la Suisse estime trop élevées. L'Inde n'autorise par exemple que l'importation des montres haut de gamme (de plus de 1000 dollars) en provenance de la Suisse. Les entreprises horlogères indiennes se plaignent de leur côté d'un accès très limité dans les foires commerciales suisses. Le dossier de la propriété intellectuelle constitue un autre domaine de divergence, car l'Inde désire profiter pleinement du délai accordé aux pays en voie de développement pour l'application des accords de l'Uruguay Round (rythme d'adaptation jugé trop lent par certains pays industrialisés).

La commission économique mixte indo-suisse, qui s'est réunie en décembre 1996, permet des échanges de vues sur les relations économiques entre les deux pays (délégation suisse menée par l'ambassadeur Nicolas Imboden, de l'OFAEE, et composée de représentants de l'économie suisse). 
En juillet 1997, la Suisse a entamé devant l'OMC une procédure de règlement du différend avec l'Inde à propos des restrictions quantitatives appliquées par l'Inde à l'importation de produits agricoles, de produits textiles et de produits industriels. Des consultations semblables ont été demandées par les Etats-Unis, l'Australie, le Canada, la Nouvelle-Zélande et l'Union européenne. Les EtatsUnis et l'Union européenne ont aussi entamé une procédure de règlement des différends dans le domaine de la protection conférée par un brevet pour les produits pharmaceutiques et les produits chimiques pour l'agriculture.

L'Inde représente toutefois un marché plein d'avenir pour l'industrie suisse d'exportation: besoins importants dans les domaines de l'infrastructure, de l'énergie et des transports, existence d'une classe moyenne indienne ayant un pouvoir d'achat élevé (la classe moyenne constitue une faible proportion de la population indienne, mais importante en nombre absolu). Les industriels suisses regrettent que le rythme de libéralisation de l'économie ne soit pas plus rapide ${ }^{17}$ et que certains secteurs restent très fermés à l'extérieur (horlogerie, textiles, banques, assurances). Le volume du commerce entre les deux pays reste encore modeste par rapport à l'importance du marché, mais les exportations de la Suisse vers l'Inde sont en forte progression (exportations de 666 millions de francs en 1996, contre 393 millions de francs en 1990). La Suisse importe principalement des textiles et vêtements ( $52 \%$ des importations, surtout des vêtements, articles de cuir, fils et tissus de coton), des produits chimiques intermédiaires, des éléments pour machines textiles, du café et des diamants. Les principaux produits suisses exportés vers l'Inde sont les locomotives et autres véhicules pour voies ferrées (157 millions de francs en 1996), des machines textiles et de filage ainsi que des produits chimiques, des médicaments et des colorants (29 millions de francs).

La Suisse occupe le huitième rang des investisseurs étrangers en Inde (avec un montant de 400 millions de dollars investis de 1991 à 1995). Parmi les investissements récents, on peut signaler la délocalisation en Inde du traitement informatique de la billetterie de Swissair. Swiss Telecom a investi 215 millions de dollars en 1996 pour s'imposer sur le marché indien du téléphone mobile. L'Inde est le pays asiatique où Asea Brown Boveri est le plus fortement implanté (ABB y emploie 10'500 personnes et réalise un chiffre d'affaires d'environ 1,5 milliard de francs). Parmi les entreprises suisses présentes en Inde, on peut mentionner Nestlé, Hürlimann (bières), Bally, Novartis, Benninger (machines de tissage), Sulzer (machines textiles) et Lista (meubles) ${ }^{18}$.

CDTM 98, voir aussi la partie «Analyses et positions» concernant la position de l'Inde et de la Suisse dans le domaine de la protection des droits de propriété intellectuelle, avec notamment le cas du secteur des médicaments.

\section{- Laos}

Un accord de protection des investissements a été conclu le 4 décembre 1996. Le commerce Suisse-Laos est très modeste: exportations suisses de 0,8 million de francs et importations de 1,4 million de francs en 1996 (importations de vêtements).

17. Selon une enquête menée par le Vorort auprès des industriels suisses. Vorort, Schweizerischer Handels- und IndustrieVerein, Wirtschaftspolitisches Jahrbuch 1996, Zurich, SHIV, avril 1997, p. 65. Voir également Neue Zürcher Zeitung, 27. November 1997 «Zögernder Abbau von Indiens Handelsschranken: Dehli unter Druck des WTO-Regimes».

18. Pour les investissements suisses en Inde, voir aussi: Arbeitsgemeinschaft der Hilfswerke. Dokument Privatwirtschaft und Entwicklung: Direktinvestitionen in Indien (Kurzfassung einer Studie von Göpf Berweger), Nr. 3, Bern, Juli 1997, 32 p. 


\section{- Philippines}

Un accord pour la protection et la promotion des investissements a été signé le 31 mars 1997, lors de la visite de Jean-Pascal Delamuraz dans ce pays, à la tête d'une délégation économique. Le Conseiller fédéral a aussi souhaité que les négociations sur la convention de double imposition puissent aboutir rapidement. Il s'est également rendu au siège de la Banque asiatique de développement.

Les exportations de la Suisse vers les Philippines s'élevaient en 1996 à 247,3 millions de francs (surtout machines et électronique, produits pharmaceutiques et insecticides). Les importations en provenance des Philippines s'élevaient à 55,4 millions de francs (surtout produits alimentaires et fruits, vêtements et chaussures, pierres synthétiques, circuits intégrés). La Suisse figure parmi les dix pays principaux investisseurs aux Philippines.

\section{- Thaïlande}

La convention de double imposition avec la Thaïlande a été signée le 12 février 1996. Le message a été adopté en septembre 1996 par le Conseil des Etats.

Un accord de protection des investissements a été signé le 17 novembre 1997, à l'issue de la visite d'Arnold Koller. Si les grandes entreprises suisses sont déjà présentes en Thaïlande depuis longtemps, la Suisse souhaite par cet accord encourager les PME à investir dans ce pays. La Thaïlande est le cinquième marché d'exportation pour la Suisse en Asie, avec un volume d'exportation de 895 millions de francs en 1996 (552 millions de produits importés de Thaïlande la même année). Ce pays est un marché important pour les exportations suisses de machines et d'électronique, de produits horlogers et bijoutiers, de médicaments et d'autres produits chimiques. Avec plus de 110'000 touristes suisses en 1996, la Thailande est le premier pays de destination en Asie pour les touristes suisses devant Hongkong (70'000 touristes suisses), Singapour $\left(61^{\prime} 000\right)$ et Israël $\left(44^{\prime} 000\right)^{19}$. La Suisse est le neuvième pays plus grand investisseur étranger, avec des investissements de près d'un milliard de francs ${ }^{20}$. La Thaïlande traverse une grave crise financière (chute de la monnaie locale, effondrement de la Bourse, institutions financières au bord de la banqueroute).

Arnold Koller a aussi signé à cette occasion un traité permettant aux prisonniers suisses de purger la fin de leur peine dans leur pays d'origine (14 Suisses sont actuellement emprisonnés en Thaïlande).

\section{- Turquie}

Le conseiller fédéral Jean-Pascal Delamuraz a entrepris un voyage en Turquie en mai 1997. La Suisse aimerait conclure une convention de double imposition et elle estime que la Turquie est en retard dans le domaine de la protection de la propriété intellectuelle relativement aux secteurs chimique et pharmaceutique. La question des droits de l'Homme a aussi été abordée. C'était la première visite officielle de la Suisse depuis les événements du 24 juin 1993, date à laquelle un manifestant kurde avait été abattu par un garde turc devant l'ambassade de Turquie à Berne.

La présence d'entreprises suisses en Turquie est importante. Parmi elles, on trouve Asea Brown Boveri (qui a équipé le barrage d'Atatürk), Novartis, Roche,

19. Source des données sur le tourisme: Les touristes suisses à l'étranger, Berne, Office fédéral de la statistique, série Tourisme, $\mathrm{n}^{\circ} 10,1997$.

20. L'Agefi, 18 novembre 1997 «Accord bilatéral signé entre la Suisse et la Thaïlande». 
Danzas, Eternit, Landis \& Gyr, Nestlé, Rieter, Saurer, SGS, Sulzer et Swissair ${ }^{21}$. Avec 71'000 Suisses partant en vacances en Turquie, ce pays était en 1996 le $15^{\circ}$ pays de destination le plus important des touristes suisses.

Les relations commerciales avec la Turquie sont intenses. Les exportations de la Suisse vers la Turquie se sont élevées à 1,145 milliard de francs en 1996 (importations de 275 millions de francs). Plus de la moitié d'entre elles concerne les machines et l'électronique (32\% de produits chimiques et $7 \%$ de produits horlogers et bijoux).

\section{a Vietnam}

La convention de double imposition a été signée en 1996 et approuvée par le Conseil des Etats en mars 1997 et par le Conseil national en juin 1997. Plusieurs facteurs peuvent inciter les investisseurs étrangers à s'intéresser de plus en plus au Vitenam: nouvelle législation libérale sur les investissements, potentiel considérable du marché intérieur, attrait du marché du travail, situation géographique en Asie, levée de l'embargo américain sur le commerce. Fin 1995, la Suisse figurait au $11^{\mathrm{e}}$ rang des investisseurs étrangers au Vietnam, avec des investissements cumulés de 580 millions de dollars (juste derrière la France). ABB, Nestlé, Novartis et Holderbank sont présents sur ce marché. Les exportations de la Suisse vers ce pays s'élevaient à 74 millions de francs en 1996 (surtout des machines, turboréacteurs et propulseurs, insecticides, fongicides et herbicides), alors que les importations en provenance du Vietnam s'élevaient à 46 millions de francs (chaussures, vêtements et maroquinerie). Le Vietnam est dorénavant un pays de concentration de l'aide suisse.

En 1997, la Suisse et le Vietnam ont paraphé un accord sur la protection de la propriété intellectuelle et la coopération. C'est le premier accord orienté exclusivement sur le domaine de la propriété intellectuelle, même si les accords de libreéchange conclus entre l'AELE et des pays tiers ou les accords bilatéraux de commerce comprennent souvent un volet sur la propriété intellectuelle. Cet accord prévoit que le Vietnam doit assurer un niveau de protection de la propriété intellectuelle équivalent aux accords TRIPS (Accord sur les aspects des droits de propriété intellectuelle qui touchent au commerce - ADPIC) de l'OMC dans un délai de deux ans après l'entrée en vigueur de l'accord (au lieu du délai plus long toléré par les accords TRIPS). Un programme de coopération technique doit aider le Vietnam à s'adapter à l'accord de l'OMC: renforcement du cadre juridique et législatif, appui à l'administration, encouragement à la mise en œuvre des lois et garantie de leur respect.

ASTM 98, dans le dossier «Analyses et positions» sur la propriété intellectuelle, l'article de Thu-Lang Tran détaille le contenu de l'accord conclu entre la Suisse et le Vietnam.

\section{Amérique latine}

Le conseiller fédéral Flavio Cotti a entrepris un voyage en Amérique latine du 21 au 26 avril 1997, au Pérou, en Argentine et au Brésil, avec des collaborateurs de la DDC et des représentants de l'économie privée. Le Conseiller fédéral a salué

21. Sur les relations Suisse-Turquie, voir: Marina Widmer, Die Türkei drängt nach Europa und der Westen braucht die Türkei - Studie über die Finanz- und Wirtschaftsbeziehungen zwischen der Schweiz und die Türkei, hrsg. AFP Aktion Finanzplatz 1996, 74 p. L'étude peut être commandée à l'Action Place financière Suisse-Tiers Monde, tél. $031 / 311.76 .16$. 
le fait que ces pays se sont engagés dans un processus de réformes et de libéralisation de leur économie, ce qui constitue une condition indispensable pour une reprise des flux d'investissements.

Pour la Suisse, les principaux marchés d'exportation en Amérique latine sont le Brésil (exportations de 908 millions de francs en 1996), le Mexique (449 millions de francs), l'Argentine (339 millions), la Colombie, le Panama et le Chili.

\section{- Argentine}

Une convention de double imposition sur les revenus et la fortune a été signée le

23 avril 1997 lors de la visite de Flavio Cotti en Argentine. Les négociations avaient été entamées en 1979 déjà, puis interrompues pour des raisons politiques et économiques, avant de reprendre en 1993. Le conseiller fédéral a aussi inauguré l'Expo Suiza, foire économique à laquelle ont participé 80 entreprises suisses d'exportation (dont deux tiers sont présentes en Argentine par des filiales ou des représentations locales). L'exposition a été organisée par l'OSEC et la Chambre de commerce Suisse-Argentine, avec une contribution financière de la Confédération. Les investissements suisses en Argentine s'élevaient à 650 millions de dollars fin 1995 et représentaient quelque 10'000 emplois ( $11^{\mathrm{e}} \mathrm{rang}$ des principaux investisseurs étrangers). C'est le troisième partenaire économique de la Suisse en Amérique latine. Les investissements sont diversifiés: on en trouve dans les secteurs des machines, de la construction, des produits pharmaceutiques et chimiques, de l'alimentaire, des services, des assurances et des banques.

\section{Brésil}

Des rencontres ont eu lieu en avril 1997 avec les milieux d'affaires ainsi que les ministres des Finances et du Budget. Le Brésil est le principal pays partenaire de la Suisse en Amérique latine (exportations de la Suisse de 908 millions de francs en 1996, importations de 283 millions de francs). Les principaux groupes de produits exportés par la Suisse sont les produits chimiques (54\% des exportations vers ce pays), les machines et l'électronique (29\%) et les produits horlogers $(8 \%)$. Les importations en provenance de ce pays sont surtout des produits agricoles (55\% des importations). La Suisse reste le cinquième plus grand investisseur au Brésil, mais son influence tend à reculer (troisième investisseur étranger jusqu'en 1980, puis devancé par les Britanniques et le Japon). Les grandes entreprises suisses sont bien présentes sur les marchés brésilien et argentin, mais il reste un grand potentiel inexploité pour les $\mathrm{PME}^{22}$.

\section{- Pérou}

Une délégation suisse mixte s'est rendue au Pérou du 6 au 10 novembre 1996. Elle était menée par Nicolas Imboden (OFAEE) et comprenait des représentants de l'économie (industrie chimique et des machines, horlogerie, industrie du ciment et banques). La mission a débouché sur la signature d'un accord de coopération entre la Suisse et le Pérou, pour intensifier la coopération économique dans les domaines commerciaux, des investissements et du transfert de technologies. Les échanges commerciaux entre la Suisse et le Pérou sont relativement modestes et en baisse ces dernières années, avec des exportations de 52,7 millions de francs en 1996 (contre 82 millions en 1994), alors que les importations de la Suisse en provenance de ce pays s'élevaient à 12,5 millions de francs

22. Sur les relations Suisse-Brésil, voir: Serge Ghinet et Gérard Perroulaz, Comment mieux coopérer avec le Brésil? Aide des ONG et relations économiques de la Suisse avec le Brésil, Genève, IUED, 199558 p. 
(16 millions en 1994). Les principaux produits exportés en 1996 ont été les machines, les appareils électriques ainsi que des produits chimiques et pharmaceutiques. La Suisse importait essentiellement des produits alimentaires et du café, des textiles (fils de coton) et vêtements, et des machines. Plusieurs grandes entreprises suisses sont présentes au Pérou (Nestlé, ABB, Novartis, Roche, Sulzer, Schindler, SGS, SBS... $)^{23}$.

Flavio Cotti a signé un traité d'entraide judiciaire avec le Pérou le 21 avril 1997. L'accord doit faciliter l'entraide judiciaire pour lutter contre le trafic de drogues, le blanchiment de capitaux et les délits fiscaux. C'est le deuxième accord de ce type en Amérique latine, après celui conclu avec l'Equateur. Lors du voyage, le Conseiller fédéral et Walter Fust ont aussi visité des projets de coopération. Le Pérou est un pays de concentration de l'aide. Il a notamment bénéficié de mesures de désendettement, avec création d'un fonds de contrepartie qui a pour objectif de financer des projets de développement dans les secteurs de l'infrastructure sociale et de la protection de l'environnement. Un accord de protection des investissements entre les deux pays est en vigueur depuis novembre 1993. Un accord commercial pour stimuler les échanges et resserrer les liens économiques existe déjà depuis 1953.

CI ASTM 97, «Entraide judiciaire internationale», pp. 98-99.

\section{- Venezuela}

Une convention de double imposition a été signée le 20 décembre 1996 avec le Venezuela. Le message a été approuvé par le Conseil des Etats en juin 1997 et par le Conseil national en octobre 1997.

Fin 1995, la Suisse figurait au troisième rang des investisseurs les plus importants dans ce pays, derrière les Etats-Unis et les Pays-Bas ${ }^{24}$. Les exportations de la Suisse vers le Venezuela se sont élevées à 113 millions de francs en 1996, alors que les importations étaient inférieures à 9 millions de francs.

\section{Afrique}

Les principaux pays destinataires des exportations suisses en Afrique sont (par ordre décroissant) l'Afrique du Sud, l'Egypte, le Maroc et le Nigeria. La Suisse importe des produits surtout en provenance de la Libye, de l'Afrique du Sud, du Nigeria et de l'Algérie (principalement produits énergétiques, métaux précieux et matières premières).

\section{- Afrique du Sud}

L'accord de promotion et de protection réciproque des investissements signé en juin 1995 est entré en vigueur le 29 novembre 1997. Par cet accord, les deux pays souhaitent garantir une meilleure sécurité juridique aux investisseurs et améliorer les conditions-cadres favorables aux investissements étrangers ${ }^{25}$. La Suisse est le sixième investisseur étranger en Afrique du Sud.

23. Voir notamment l'article de La Vie économique de décembre 1995 «L'économie péruvienne - Présence de la Suisse au Pérou".

24. L'Agefi, 11 juin 1997. Voir aussi La Vie économique de mars 1997 «Le Venezuela - Une économie en pleine mutation».

25. Pour les relations entre la Suisse et l'Afrique du Sud, voir: Mascha Madörin, Dossier Südafrika - Weltmarkt und die Schweiz. Die wirtschaftsbeziehungen der Schweiz mit Südafrika in den 90er Jahren, NaSA, Nachrichtenstelle Südliches Afrika, 74 p., à commander à l'Action Place financière Suisse-Tiers Monde, tél. 031/311.76.16. 
Le président sud-africain, Nelson Mandela, est venu pour la première fois en visite officielle en Suisse du 2 au 6 septembre 1997. Les discussions ont porté sur les relations économiques bilatérales et la coopération technique suisse en Afrique du Sud.

Les exportations de la Suisse vers l'Afrique du Sud s'élevaient à 696 millions de francs en 1996 (surtout produits chimiques, machines et électronique et métaux précieux). Les importations en provenance de l'Afrique du Sud s'élevaient à 360 millions de francs (dont deux tiers de métaux précieux et de pierres gemmes et $22 \%$ de produits agricoles).

\section{Europe centrale et orientale}

\section{- Croatie}

Un accord de protection des investissements a été signé en automne 1996.

\section{- Slovaquie}

Une convention de double imposition a été signée en février 1997 (une convention semblable avait été signée en 1995 avec la Tchéquie), puis adoptée par le Conseil des Etats en octobre 1997.

Même si les montants sont encore modestes, la Suisse figurait en 1994 parmi les dix plus importants investisseurs en Slovaquie. Les exportations de la Suisse vers la Slovaquie s'élevaient à 140 millions de francs en 1996 (exportations de 546 millions vers la Tchéquie).

\section{- Slovénie}

Un accord de protection des investissements a été signé en novembre 1995, puis une convention de double imposition le 12 juin $1996^{26}$. Le Conseil des Etats a approuvé cette convention en mars 1997 et le Conseil national a donné son accord en juin 1997. Il existe en outre un accord de libre-échange entre la Slovénie et les Etats de l'AELE.

La Slovénie est l'Etat issu de l'ex-Yougoslavie avec lequel la Suisse entretient les relations commerciales les plus importantes, en forte augmentation depuis 1992. Les exportations de la Suisse vers la Slovénie se sont élevées à 186 millions de francs en 1996 (importations de 86 millions de francs la même année). Pour comparaison, les exportations de la Suisse vers la Serbie se sont élevées à 97 millions de francs en 1996, celles vers la Croatie à 79 millions de francs et celles vers la Macédoine à 77 millions de francs ( 16 millions vers la Bosnie). La Suisse était en 1993 le cinquième investisseur le plus important en Slovénie, même si ces investissements sont encore modestes. 


\section{SOURCES}

« Message sur la prorogation de l'arrêté fédéral concernant la conclusion de traités relatifs à la protection et à l'encouragement des investissements de capitaux » du 20 octobre 1993, Berne, message 93.086.

« Message concernant une convention de double imposition avec la République de Slovénie » du 16 octobre 1996, Berne, message 96.065 .

« Message concernant une convention de double imposition avec le Venezuela » du 10 mars 1997, Berne (in Feuille fédérale, $\mathrm{n}^{\circ} 17$, vol. II, 6 mai 1997, pp. 1028-1057, message 97.026).

«Message concernant une convention de double imposition avec la République slovaque » du 28 mai 1997, Berne (in Feuille fédérale, $\mathrm{n}^{\circ} 27$, vol. III, 15 juillet 1997, pp. 989-1014, message 97.044).

« Message concernant une convention de double imposition avec l'Argentine » du 13 août 1997, Berne (in Feuille fédérale, $\mathrm{n}^{\circ} 37$, vol. IV, 23 septembre 1997, message 97.057).

Union suisse du commerce et de l'industrie, Annuaire 1995 de politique économique, Zurich, Vorort, mars 1996.

Schweizerischer Handels- und Industrie-Verein, Wirtschaftspolitisches Jahrbuch 1996, Zürich, SHIV, April 1997.

L'Agefi, 3 avril 1997, 21 avril 1997, 24 avril 1997, 12 mai 1997, 11 juin 1997, 18 novembre 1997 «Accord bilatéral signé entre la Suisse et la Thaïlande».

Journal de Genève, 6 décembre 1996 (réunion de la commission mixte indo-suisse), 2 avril 1997 «J.-P. Delamuraz en visite aux Philippines», 5-/6 avril 1997 «Visite fructueuse de J.-P. Delamuraz en Inde», 19-20 avril 1997 «Les intérêts économiques de la Suisse font courir Flavio Cotti en Amérique latine», 12 mai 1997 «Les relations entre la Suisse et la Turquie se réchauffent», 2 décembre 1997 «Le Vietnam veut devenir un partenaire présentable. La Suisse s'y emploie».

La Liberté, ${ }^{\text {er }}$ avril 1997 «Delamuraz a signé un accord bilatéral aux Philippines», 10-11 mai 1997 «JPD veut redynamiser les relations turco-suisses», 2 décembre 1997 «Au Vietnam, la Suisse expérimente une nouvelle aide: changer les têtes".

Neue Zürcher Zeitung, 1. April 1997 «Jean-Pascal Delamuraz trifft Fidel Ramos», 5.-6. April 1997 «Abschluss des Besuchs von Delamuraz in Indien», 16. April, 22. April 1997 «Bundesrat Cotti auf Promotionstour in Peru», 24. April 1997 «Argentinien-Besuch Cottis im Zeichen der Wirtschaft», 28. April 1997 «Positive Bilanz von Cottis Südamerikareise», 10.-11. Mai 1997 «Bundesrat Delamuraz in der Türkei», 4. September 1997 «Offizieller Besuch Nelson Mandelas in Bern», 10. Oktober 1997, 18. November 1997 «Thailand letzte Station von Kollers Asienreise», 27. November 1997. 